\title{
Quantum Creation of Closed Universe with Both
Effects of Tunneling and Well
}

\section{De-Hai Zhang}

\author{
(e-mail: dhzhang@sun.ihep.ac.cn) \\ Department of Physics, Graduate School in Beijing, \\ University of Science and Technology of China, \\ P.O.Box 3908, Beijing 100039, P.R.China.
}

\begin{abstract}
:
A new "twice loose shoe" method in the Wheeler-DeWitt equation of the universe wave function on the cosmic scale factor $a$ and a scalar field $\phi$ is suggested in this letter. We analysis the both affects come from the tunneling effect of $a$ and the potential well effect of $\phi$, and obtain the initial values $a_{0}$ and $\phi_{0}$ about a primary closed universe which is born with the largest probability in the quantum manner. Our result is able to overcome the "large field difficulty" of the universe quantum creation probability with only tunneling effect. This new born universe has to suffer a startup of inflation, and then comes into the usual slow rolling inflation. The universe with the largest probability maybe has a "gentle" inflation or an eternal chaotic inflation, this depends on a new parameter $q$ which describes the tunneling character.
\end{abstract}

\section{Introduction}

A lot of observations have proved that our universe undergoes a big bang ${ }^{[1]}$ from a high temperature and high density state. Today our universe is very large and old, very isotropic and homogeneous in the large scale, and almost flat. In order to illustrate all of these features, our universe in its very early period needs a more violence expansion, i.e., inflation ${ }^{[2]}$ which must has an enough large inflating e-folds and a small background fluctuation. But somebody would like to ask what is before the inflation? Why did the universe inflate? Further he could ask whether the universe had its birth? How was the universe born? These are very interesting problems the wise mankind want to probe into. Some scientists have supplied for us a part of possible answers about these questions by right of their insights. The universe may be born in quantum way ${ }^{[3]}$, and it may suffer eternal chaotic inflation ${ }^{[4]}$. Maybe it is born from an instanton ${ }^{[5]}$. However, the present theories have still some difficulties, many details are still unclear.

We all hope that a theory will be simple as possible as. However for a most typical potential, such as $m^{2} \phi^{2} / 2$, in chaotic inflation theory, it meets some difficulty which 
can be seen by following analysis. At first there is a famous probability formula about the quantum birth of the universe, $\rho \propto \exp \left[-24 \pi^{2} M_{p}^{4} V^{-1}(\phi)\right]$, in the quantum cosmology ${ }^{[6]}$, where $8 \pi G=M_{p}^{-2}=\kappa^{2}$, and $M_{p}=2.4 \times 10^{18} \mathrm{GeV}$ is the Planck mass. If this formula is earnest, the largest probability happens at a place where the field value $\phi$ is largest, then maybe it tends to infinity. However if $\phi$ exceeds over a critical value, the universe will undergo an excessive inflation and never roll down. Or we choose a special potential, or we need a cutting off or a suppression of the probability at large field limit. We call it as "large field difficulty".

In this paper we find out that a potential well effect appeared naturally in the quantum cosmology can overcome this difficulty. My starting point is still the famous Wheeler-DeWitt equation ${ }^{[7]}$ of the universe wave function on the cosmic scale factor $a$ and a scalar field $\phi$. As a difference from the former investigations, I study both quantum behaviors about $a$ and $\phi$, i.e., a tunneling effect for $a$ and a potential well effect for $\phi$ (the latter is new for me). Just this well effect supply a probability suppression of large field limit. Just these two combined effects of the tunneling and the well determine that a primary universe has to have a most possible initial state, which initial values may be suitable to develop a relevance universe for us through the startup of its inflation. If this initial field is not too large, the universe maybe doesn't have an eternal chaotic inflation, therefore this probability makes sense.

In order to illustrate my idea and results, at first we distinguish three inflationary states in the section 2. Then we review the tunneling effect and show "large field difficulty" in the section 3. We study the well effect and introduce an important parameter $q$ to describe the tunneling character in the section 4. The startup, persistent and ending of inflation are studied in the section 5. We give a more discussion about our results in the section 6 . Finally we point out its progress and shortcoming of our method in the last section.

\section{Gentle, chaotic and excessive inflations}

Among various scenarios to describe the birth and evolution of the universe, the eternal chaotic inflation is an important one. The necessary condition at which the eternal chaotic inflation happens is that the initial field value $\phi_{0}$ of inflaton is near a critical one $\phi_{c}$ (index $c$ means critical). This critical value is determined by the condition that the average quantum fluctuation $\Delta \phi_{q u}$ is near the classical rolling down value $\Delta \phi_{c l}$ of field in a Hubble time. In the case of potential $m^{2} \phi^{2} / 2$, which is regarded as a typical one for chaotic inflation and is studied mainly in this paper, this critical value is ${ }^{[8]}$

$$
\phi_{c}=2 \cdot 6^{1 / 4} \pi^{1 / 2} \kappa^{-3 / 2} m^{-1 / 2},
$$

which is depend on the mass parameter heavily.

Let us estimate rough mass at first. The constraint on mass comes from the small fluctuation about $10^{-5}$ of the Cosmic Microwave Background Radiation (CMBR $)^{[9]}$,

$$
\delta^{2}=150^{-1} \pi^{-2} \epsilon_{e}^{-1} \kappa^{4} V_{e}=10^{-10},
$$


where $\epsilon_{e} \simeq 1$ (index $e$ means ending of inflation) is the slow rolling parameter $\epsilon=\kappa^{-2} V^{\prime 2} V^{-2} / 2$ of the ending point of inflation. From it we obtain the mass parameter $m \simeq 4 \times 10^{-4} \kappa^{-1} \simeq 10^{15} \mathrm{GeV}$ or little small, which is just about the grand unification energy scale. Then the field critical value of chaotic inflation is $\phi_{c}=280 \kappa^{-1}$ and the potential is $V_{c}=6 \times 10^{-3} \kappa^{-4}$. We think that up to $V_{q} \simeq \kappa^{-4}$, i.e., $\phi_{q} \simeq 3500 \kappa^{-1}$ (index $q$ means quantum), the classical gravity maybe should be applicable.

The eternal chaotic inflation is not the unique possible case. When $\phi_{0} \ll \phi_{c}$, it is obvious that the eternal chaotic inflation can not happen due to the quantum fluctuation too small, but it has a normal inflation if $\phi_{0} \geq \phi_{e}$ where $\phi_{e}$ is the field value at the ending of inflation, which is determined by the end of slow rolling $\epsilon_{e} \simeq 1$ and gets $\phi_{e}=\sqrt{2} \kappa^{-1}$ for potential $V=m^{2} \phi^{2} / 2$. We call it as "gentle" or "classical" inflation. When $\phi_{0} \gg \phi_{c}$, a tiny classical rolling down is submerged in a huge quantum fluctuation of $\phi$, then in spite of the universe undergoes an eternal chaotic inflation, but it can roll down never and nowhere, any slow rolling down universe is not produced in its fraction. Maybe the field value can climb higher and higher. We call this case as "excessive" or "quantum" inflation. Of course we only call the case $\phi_{0} \approx \phi_{c}$ (maybe its range is rather wide) as standard "chaotic" or "critical" inflation. Therefore the chaotic inflation which can produce an observed universe is conditional.

\section{The tunneling effect of scale factor}

The birth of the universe is a complicated problem. If we predigest this problem to freeze out all of freedoms except the cosmic scale factor $a$ and a scalar field $\phi$, we can obtain the famous Wheeler-DeWitt equation ${ }^{[7]}$

$$
\frac{\partial^{2} \psi}{\partial a^{2}}-\frac{6}{\kappa^{2} a^{2}} \frac{\partial^{2} \psi}{\partial \phi^{2}}-\frac{144 \pi^{4}}{\kappa^{4}}\left(K_{c} a^{2}-\frac{\kappa^{2}}{3} a^{4} V(\phi)\right) \psi=0
$$

where $\psi$ is the wave function of the universe and $V(\phi)$ is a potential of this scalar field. $K_{c}$ is the sign of the curvature term. This is intricate equation, and it is hard to explain the meaning of the wave function. In order to obtain a meaningful result a simplifying method is needed. We adopt a new so-called "twice loose shoe" $\operatorname{method}^{[10]}$, i.e., to fix a variable and to let another variable vary respectively. When one fixes at first step the scalar field $\phi$, i.e., $\partial \psi / \partial \phi=0$, one has the equation on $a$ from Eq.(2)

$$
\frac{\partial^{2} \psi}{\partial a^{2}}=\frac{144 \pi^{4}}{\kappa^{4}}\left(K_{c} a^{2}-\frac{\kappa^{2}}{3} a^{4} V(\phi)\right) \psi
$$

This is a following standard problem of non-relativity quantum mechanics,

$$
H \psi=E \psi, \quad H=-\frac{\hbar^{2}}{2 m} \frac{\partial^{2}}{\partial x^{2}}+U(x), \quad \frac{\partial^{2} \psi}{\partial x^{2}}=\frac{2 m}{\hbar^{2}}(U(x)-E) \psi
$$

We know that Eq.(3) is a tunneling problem. Which potential and total energy are 


$$
U(a)=\frac{144 \pi^{4}}{\kappa^{4}}\left(K_{c} a^{2}-\frac{\kappa^{2}}{3} a^{4} V(\phi)\right), \quad E_{a}=0 .
$$

We note that only for $K_{c}=1$ the potential can form a barrier and has a quantum tunneling effect. In this case the universe created is closed one. In other hand, it is very hard to imagine that a flat or open universe with real infinite volume can be created immediately from nothing by means of any process. Therefore we suppose that only a finite closed universe can be produced by quantum tunneling effect. $U(a)$ is a potential barrier, a "particle" will tunnel through it, and the tunneling probability is a standard result ${ }^{[6]}$

$$
\rho_{a}=c_{1} \exp \left(-\frac{3 \pi}{G \Lambda}\right)=c_{1} \exp \left(-\frac{24 \pi^{2}}{\kappa^{4} V(\phi)}\right) .
$$

Note that it is independent on $a$. Here we don't debate the "sign problem", we think this sign is more reasonable.

Serious problem is that there exists a so-called "large field difficulty" for this probability formula which statement is that if the potential is a monotonous rising unbounded one, such as $m^{2} \phi^{2} / 2$, then the larger the $V$ is, the higher the probability is. Therewith the initial field value $\phi_{0}$ maybe tend towards infinity (at least possible up to value $\phi_{q}$ mentioned above), and the primary universe perhaps stays in state of the excessive inflation. The key is that the most possible $\phi$ value is not adjustable, which takes always its the largest one as possible as. In order to obtain a suitable eternal chaotic inflation we have to choose a special potential (undulate one?), or to cut off the applicable range of the tunneling probability formula in case of unbounded potentials. Otherwise we can not avoid this large field difficulty for this simple potential.

\section{The potential well effect of the scalar field}

After the tunneling, the $a$ takes transition from $a=0$ to a non-zero value $a_{0}$ determined by the equation $U\left(a_{0}\right)=0$ of the turning point,

$$
\kappa^{2} a_{0}^{2} V\left(\phi_{0}\right)=3
$$

i.e., if we know $\phi_{0}$ of the primary universe, then we can calculate its $a_{0}$.

When we use the loose shoe method at second step to fix the cosmic scale factor $a$, i.e., $\partial \psi / \partial a=0$, we obtain the equation on $\phi$ from Eq.(2)

$$
\frac{\partial^{2} \psi}{\partial \phi^{2}}=8 \pi^{4} a^{6}\left(V(\phi)-\frac{3}{a^{2} \kappa^{2}}\right) \psi
$$

Comparing with the standard equation of quantum mechanics, it is a deep well problem clearly, and its potential and total energy are

$$
U(\phi)=8 \pi^{4} a^{6} V(\phi), \quad E_{\phi}=24 \pi^{4} \kappa^{-2} a^{4} .
$$


Remember that the $a$ is fixed here, therefore it doesn't obey a similar equation $\kappa^{2} a^{2} V(\phi)=3$ with Eq.(5), otherwise the Eq.(6) will be trivial. What value the $a$ can be fixed to? Let us do an analysis. Before tunneling, nothing exits $a=0$, then $U(\phi)$ and $E_{\phi}$ all vanish, there is not any potential well for $\phi$. After tunneling, the cosmic scale factor has been $a_{0}$ suddenly, in this time the well is the most steep. In an actual process, the $a$ undergoes a tunneling, we can imagine that $a$ changes from 0 to $a_{0}$ in the fictive process of the tunneling, therefore it is reasonable to suppose that $a$ takes a middle value in the classical forbidden range between 0 and $a_{0}$, i.e., $a=q a_{0}$, and an important parameter $q$ is introduced here which is a number factor and is less than 1 .

When the potential is a square power one, i.e., the mass term $V=\frac{1}{2} m^{2} \phi^{2}$, this is a harmonic oscillation problem and the Eq.(6) becomes as

$$
\frac{\partial^{2} \psi}{\partial \phi^{2}}=\left(4 \pi^{4} q^{6} a_{0}^{6} m^{2} \phi^{2}-\frac{24 \pi^{4}}{\kappa^{2}} q^{4} a_{0}^{4}\right) \psi
$$

We can do a variable transformation $\phi=s y$ to obtain the standard form

$$
\frac{\partial^{2} \psi}{\partial y^{2}}=\left(y^{2}-\lambda\right) \psi, \quad s^{-2}=2 \pi^{2} q^{3} a_{0}^{3} m, \quad \lambda=2 n+1=\frac{12 \pi^{2} q a_{0}}{\kappa^{2} m} .
$$

The Eq.(7) has the solution

$$
\psi_{\phi}=c_{2} \exp \left(-\pi^{2} q^{3} a_{0}^{3} m \phi^{2}\right) \cdot w_{n}(\phi)
$$

where $w_{n}(\phi)$ is a polynomial for which the leading power term is $\phi^{n}$. We shall see that only this highest term is important for our later analysis since we want merely to consider a case of the larger values of field $\phi$ or $y$ and power $n$.

Let us now consider the both effects comes from the tunneling and the well. If unexpected complication is not involved, one can envisage that a combined probability should be

$$
\rho=\psi_{\phi}^{2} \rho_{a}=c_{1} c_{2}^{2} \exp \left(2 n \ln (\kappa \phi)-2 \pi^{2} q^{3} a_{0}^{3} m \phi^{2}-\frac{48 \pi^{2}}{\kappa^{4} m^{2} \phi^{2}}\right) \propto \exp (F(\phi)) .
$$

We see that just the well effect supplies a powerful suppression of this probability for large $\phi$ value. This is what we hope in order to solve the large field difficulty. The largest probability happens at the point $F^{\prime}\left(\phi_{0}\right)=0$, i.e.,

$$
\frac{2 n}{\phi_{0}}-4 \pi^{2} q^{3} a_{0}^{3} m \phi_{0}+\frac{96 \pi^{2}}{\kappa^{4} m^{2} \phi_{0}^{3}}=0 .
$$

In this time we can substitute with $n=6 \pi^{2} q \kappa^{-2} m^{-1} a_{0}$ and $a_{0}=\sqrt{6} \kappa^{-1} m^{-1} \phi_{0}^{-1}$ in according to Eq.(8) and Eq.(5) respectively, and obtain finally

$$
\phi_{0}=4 \sqrt{2 / 3} \cdot q^{-1}\left(2 q^{2}-1\right)^{-1} \kappa^{-1},
$$

i.e., the most possible inflaton field value when the universe is born in quantum manner. We see that if the parameter $q$ is near to $2^{-1 / 2}$, the $\phi_{0}$ will approach to infinity. Of course it is not able to be taken seriously due to approximation of our method. How to solve and understand truly Eq.(2) is a serious challenge! 


\section{Startup, persistent and end of inflation}

The transformation for the universe from quantum to classical needs a process. For simpleness we assume that after the universe is born by quantum manner, it comes soon into the classical evolution. However the universe does not come into inflation phase immediately due to a huge curvature term. The equation of its motion is ${ }^{[10]}$

$$
3 \kappa^{-2}\left(\frac{\dot{a}}{a}\right)^{2}=\frac{1}{2} \dot{\phi}^{2}+\frac{1}{2} m^{2} \phi^{2}-\frac{3 \kappa^{-2}}{a^{2}}, \quad \ddot{\phi}+3 \frac{\dot{a}}{a} \dot{\phi}+m^{2} \phi=0,
$$

with the initial condition $\phi_{0}$ of Eq.(12), $\dot{\phi}_{0}=0$ and $a_{0}=\sqrt{6} \kappa^{-1} m^{-1} \phi_{0}^{-1}$ at the starting time $t=0$ chosen by us. In this time the Hubble constant $\left.H\right|_{t=0}$ is zero and density ratio $\left.\Omega\right|_{t=0} \rightarrow \infty$. At first we research how the universe begins to inflate. The startup of the inflation in this case is similar at all with the pure cosmological constant case $\Lambda=\kappa^{2} V_{\Lambda}=3 a_{0}^{-2}$. In the latter case, we have $H=$ $\dot{a} / a=a_{0}^{-1} \tanh \left(a_{0}^{-1} t\right)$, but this expression only can applicable in the first few $a_{0}$ 's of time. After this time, i.e., about $t \geq 3 a_{0}$, the Hubble constant has arrived its highest value $H_{b}$ (index $b$ means beginning of inflation), and then $H$ begins to decrease its value, and the universe comes into the normal slow rolling down and persistent inflation. This startup period of about $t_{b} \simeq 3 a_{0}$ is important to smooth whole primary universe and maybe affect the final observed universe if it is in a gentle inflation. Since $t_{b}$ is small, the difference between the original values $\phi_{0}$ of the inflaton and the values $\phi_{b}$, at which $H \approx H_{b}$ and the real inflation begins, is small. We can use the approximation $\phi_{b} \approx \phi_{0}$ to estimate the inflating e-folds. Our data simulation supported these elementary analyses. The details of this model are very rich which will be studied in our later work.

Before the parameter $q$ can be calculated exactly, we see that the most possible initial field value is adjustable if we consider both effects of tunneling and well. If $q$ is taken as $0.71 \sim 0.72$, the $\phi_{0}$ is near the $\phi_{c}$, the primary universe will evolve to an eternal chaotic inflating universe, and the primary born probability lost its meaning as explained by Guth ${ }^{[8]}$. If $q$ is taken as 0.8 , then $\phi_{0} \simeq 15 \kappa^{-1}$ and $a_{0} \simeq 400 \kappa$, the primary universe undergoes only the gentle inflation, which inflating e-fold is about $^{[9]}$

$$
N=\int M_{p}^{-2} V V^{\prime-1} d \phi=\kappa^{2}\left(\phi_{b}^{2}-\phi_{e}^{2}\right) / 4 \simeq \epsilon_{b}^{-1} / 2=53 .
$$

The concept of primary born probability is available in the gentle inflation. At the ending of inflation, the cosmic scale factor is enough large,

$$
a_{e}=e^{53} a_{b}=10^{23} a_{b}
$$

however it is still a finite closed universe, although very flat. When the inflaton rolls down to $\phi_{e}$ value, the inflaton field begins its fast oscillation, the matter particles will be produced in succession, and later on the universe comes into the radiation dominated period of the standard big bang. 


\section{More discussions about parameter $q$}

The parameter $q$ is very important in our model. Due to the tunneling effect, $q$ should be less than 1 . However if $q$ is one naively, we only have a little inflation, i.e., $\phi_{0}=3.3 \kappa^{-1}$ and $N \simeq 2$. In this case we can only put our hope on the small probability events of the universe creation. However the relative probability falls very rapidly in according to Eq.(10). This is not a way out.

We need to note that the role of the polynomial $w_{n}(\phi)$ which has an obvious affect on $\phi_{0}$. However we can know the following point that even if the largest contribution term is not the highest power term, the modification is not too remarkable. The coefficient in the exponential of the tunneling probability, in Eq.(雨), such as 24 or 12 , is also not important, but its sign is important. The main affect comes from the parameter $q$.

We can consider other potential such as $V(\phi)=\lambda \phi^{4}$, however we don't know its exact energy spectrum and are not able to estimate the effect of the polynomial in the wave function. In the same time, there is still a same problem whether the parameter $q$ has to be put in it to reflect the tunneling character.

The parameter $q$ should be able to be calculated and only is dependent on the unique model parameter, mass, if potential $m^{2} \phi^{2} / 2$ is used. Let us imagine a wonderful prospect that if we know the function $q(m)$, then we know $\phi_{0}(q(m))$. Moreover if the most possible field value $\phi_{0}$ was just the critical value $\phi_{c}(m)$ of the chaotic inflation, then we could solve the unique parameter $m$ in the model. If this mass was just one come from the CMBR, then it is an unbelievable high predicting ability for this model!

Our idea may add some important constraint on the build of the inflation models. It is not true that any potentials which can produce inflation can also suitable to the quantum birth of the universe.

Anyhow the real meaning of the total Wheeler-DeWitt equation of Eq.(2) is abstruse. We face a great challenge and opportunity.

\section{Conclusion}

The universe born probability in the quantum cosmology has its shortcoming if we only consider the tunneling effect, since the largest probability happens at a place with the largest potential. For an unbounded potential this is a disaster. The large potential has a large field value, then the universe may stay in an excessive inflating state. It is necessary to suppress the probability from the large field limit. This suppression comes just from the potential well effect which is unconsidered previously. In order to reflect the tunneling character a parameter $q$ has to be introduced, which should be able to be calculated from basic theory and only is dependent on mass parameter if a simple potential $m^{2} \phi^{2} / 2$ is used. Which state,

i.e., gentle, chaotic or excessive inflation, the universe stays in is subtly dependent on the parameter $q$. The primary universe which has zero Hubble constant has to undergo a startup period to establish its inflation, it can become smooth by means 
of this startup.

It is a pity that the ability to calculate the parameter $q$ out is absent in the present. In fact many things are not known by us. We don't know whether the "twice loose shoe" method is a proper one. We have to remember that all of methods here used are only phenomenal. We don't know why only four dimensions begins its expansion but why the extra-dimensions of the superstring or membrane without expansion. There is some speculation in our toy model. To enlighten more excellent new ideas or methods is what this paper wants to pursue.

\section{Acknowledgment:}

This work is supported by The foundation of National Nature Science of China, No.19777103. The author would like to thank useful discussions with Profs. J.R.Bond, L.Kofman, U.-L.Pen during my visiting Toronto University, and with X.-M.Zhang Y.-Z.Zhang and Y.-Q.Yu in China.

\section{References:}

[1] See e.g., P.J.E.Peebles, Physical Cosmology, Princeton University Press, Prinston, 1993.

[2] A.H.Guth, Phys.Rev.D23(1981)347.

[3] A.Vilenkin, Phys.Rev.D37(1988)888.

[4] A.Linde, Particle Physics and Inflationary Cosmology, 1990 by Harwood Academic Publishers.

[5] S.W.Hawking and N.G.Turok, Phys.Lett.B425(1998)25.

[6] A.Linde, "Quantum Creation of an Open Inflationary Universe", gr-qc/9802038.

[7] B.S.DeWitt, Phys.Rev.160(1967)1113;

J.A.Wheeler, in: Relativity, Groups and Topology, eds. C.M.DeWitt and J.A.Wheeler (Benjamin, New York, 1968).

[8] A.H.Guth, "Inflation and Eternal Inflation", astro-ph/0002156.

[9] D.H.Lyth and A.Riotto, "Particle Physics Models of Inflation and the Cosmological Density Perturbation", hep-ph/9807278.

[10] E.W. Kolb and M.S. Turner, The Early Universe, Addison Wesley, 1990. 